\title{
Optimal Multi-Channel Delivery of Expertise: An Economic Analysis
}

\author{
Zafer D. Ozdemir
}

ABSTRACT: Consumers use the Internet to minimize uncertainty when making important health-related decisions. Physicians are interested in delivering their expertise on-line even if unable to physically examine patients. A game-theoretic model was used to study the optimal channel strategies of capacity-constrained experts who provide consultation services via a face-to-face and an on-line channel. A consumer seeking consultation service can be in either a good or a bad state, each of which entails symptoms the expert can observe. As shown by the model, an expert can charge a higher price on-line than face-to-face and still find consumers willing to use the new service. If both channels are utilized, consumers who are more certain about their state are served on-line. The optimal price of an on-line consultation increases with the time required for the service. Experts need to consider several issues in pricing their services, but as the model shows, offering on-line consultations makes economic sense. Medical experts can use insights derived from the model to simultaneously manage face-to-face and on-line channels in selling their expertise.

KEY WORDS AND PHRASES: E-health, expert service, on-line consultation, private information good, telemedicine.

E-health is touted as the future of health care, promising to transform the way health care entities conduct business and to change the way patients relate to health care providers. E-health is becoming more pervasive among physicians and patients alike. The Pew Internet \& American Life Project reports that 93 million Americans had gone on-line for health information by December 2002 [17]. Six million Americans seek medical advice on the Internet every day. According to a Harris Interactive Report, 37 percent of adult Internet users in 2002 were willing to pay to communicate on-line with their physicians [34]. While consumers demand the convenience of communicating on-line with their doctors, the absence of physical examination and face-to-face dialogue may create risks for medical experts by reducing their diagnostic accuracy. Nonetheless, experts offer on-line access to their services. A study of 1,200 physicians conducted by Deloitte and Touche and Fulcrum Analytics indicated that 23 percent of physicians used e-mail to communicate with their patients in 2002 [14]. Doctors expect to be reimbursed an average of $\$ 57$ for an e-consultation. More than a dozen health insurers in several states have begun reimbursing doctors for on-line consultations with patients [35].

A recent report by the Institute of Medicine, Crossing the Quality Chasm, states that "patients should receive care whenever they need it and in many

The author thanks Jack Barron and Mark Meckler for helpful discussion, and Skip Benamati and two anonymous reviewers for their feedback and comments on earlier drafts of this paper. Greg Reese provided research support with numerical optimization.

International Journal of Electronic Commerce / Spring 2007, Vol. 11, No. 3, pp. 89-105. Copyright (C) 2007 M.E. Sharpe, Inc. All rights reserved. $1086-4415 / 2007 \$ 9.50+0.00$ DOI 10.2753/JEC1086-4415110303 
forms, not just face-to-face visits . . . access to care should be provided over the Internet, by telephone, and by other means" [11, p. 61]. Apart from the convenience, the major benefits of on-line consultations for patients include greater control over medical records, the ability to compose questions better and save e-mails to reread instructions, and a less intimidating venue due to the "relative anonymity," which allows some level of disinhibition for patients to ask questions they might not ask otherwise. Of course, emergencies and other time-sensitive issues, such as chest pain, shortness of breath, suicidality, and bleeding, cannot be dealt with effectively on-line.

There are many examples of Web sites that facilitate on-line consultations. Perhaps the most notable is RelayHealth. By July 2005, more than 4,000 physicians had listed themselves on RelayHealth's portal (http:/ / relayhealth.com) to offer e-mail consultations. The American Medical Association (AMA) lets patients search for doctors offering on-line consultations both at its own Web site (www.ama-assn.org) and at medem.com. Medem, a for-profit Internet company backed by the AMA and other medical societies, provides secure communication services that enable doctors to charge patients for on-line visits. Doctors in Medem's network reportedly charge \$26 on average for a virtual visit [8]. In addition, some of the top experts in the nation provide online second opinions for important diagnoses and treatments. The Cleveland Clinic, a well-known multi-specialty academic medical center in Cleveland, has established an on-line interface, the eClevelandClinic, to serve patients who need advice and possibly a major intervention but cannot easily access a suitable doctor in person. The electronic service is limited to life-threatening and life-altering conditions that can be safely assessed on-line, such as a new cancer diagnosis, cardiac procedures, joint replacements, and neurological problems. Patients provide a personal medical history and the original diagnosis as well as other relevant materials, such as test results, MRIs, films, x-rays, and a consent form. Three Harvard University teaching hospitals have initiated a similar service at econsults.partners.org. In addition, several leading oncologists render medical opinions at www.mdexpert.com, charging fees as high as $\$ 3,200$.

The present research investigated the optimal channel-management strategies of experts from an economic standpoint and asked the following questions: When should highly demanded medical experts offer on-line consultations regarding medical conditions that can be treated on-line? What is the optimal on-line diagnosis policy? Which types of consumers should be served faceto-face and which on-line? What is the optimal pricing policy for the two channels? The answers to these questions can guide medical providers about when to adopt and how to utilize Web-based consultation services.

The preceding questions are broadly related to three areas of research: telemedicine, multi-channel management, and expert services. Despite the recent popularity of telemedicine, researchers have not given much attention to developing a sound methodology for analyzing its cost-effectiveness with respect to alternative approaches. Instead, research on telemedicine is mainly concerned with the question "Can we do this?" and leaves "Should we do this?" unanswered [26]. Economic studies have repeatedly found that avoidance of travel or of patient transfer is a salient benefit of telemedicine, 
but most of these studies have methodological limitations, and their findings are not generalizable $[19,20]$.

The literature on multi-channel management examines how firms can utilize on-line and traditional channels simultaneously [7, 10, 22, 23, 30, 32, 36, 38]. Focused primarily on the retail sector, this stream of research seeks to understand the issues resulting from the emergence of the Internet, including the conflict between direct and indirect sales channels, management of communications strategies and consumer segmentation, impact of network externalities and switching costs, and competition with new, pure-play competitors. Important studies on electronic commerce examine the way the Internet has changed the nature of competition and the interaction between business firms and consumers in markets with differentiated product offerings [2, 4, 21, 24, 25]. Since medical experts are also confronted with the problem of managing face-to-face and on-line channels simultaneously, the studies mentioned above are relevant to the present study. It must be borne in mind, however, that consultations with experts are substantially different from retail goods.

Consultations are essentially private information goods. Until now, information systems research has examined public information goods, such as software and music, which require a significant sunk cost to produce but only a negligible cost to reproduce $[3,9,13]$. In contrast, a private information good is produced only for a specific consumer, typically with the objective of diagnosing a problem or suggesting a service to eliminate it. The economics literature on private information markets focuses on information problems that beset relationships between experts and customers in the traditional faceto-face channel. In some situations the customer may not observe the type of service provided, and this may allow the expert to defraud the customer by misrepresenting a low-cost service as a costly one [29, 37]. In other situations the customer may observe the actual service provided, but not necessarily whether the more costly service was really needed [1, 5, 12, 15, 16, 33]. Moreover, the expert's diagnostic effort may not be observable, and the success of the service may not be verifiable [28].

While the preceding information problems are important in their own right, several studies abstract away from them in the interest of isolating some feature of private information markets. For example, studies of the pricing of expert information in a duopoly market by Sarvary and by Ozdemir, Akcura, and Altinkemer showed that high-quality information sellers may specialize in second opinions [27, 31]. Bouckaert and Degryse studied the pricing and quality competition in private markets, focusing only on the face-to-face channel [6]. In a similar vein, the present paper does not emphasize the potential of fraud for the simple reason that the problem has already been discussed in the literature and the way it works is relatively well understood. Although fraud (moral hazard) is not explicitly considered, the discussion in this paper incorporates the costs associated with misdiagnosing consumer problems.

The study takes an economic perspective and contributes to telemedicine research by addressing the question of "When should we do it?" It also aims to connect the literature on multi-channel management and private information markets. The main contribution to the literature is the explicit consideration of optimal diagnosis policies, pricing strategies, and profitability when 
consulting face-to-face and on-line. The implications for highly demanded experts seeking to manage the two channels simultaneously are derived and discussed. This is accomplished by setting up a game-theoretic model that derives the value of a consultation for consumers and the expected cost of diagnostic errors for experts. Next, expressions are obtained for simultaneously determined optimal channel and diagnostic strategies, and the optimal pricing strategies of experts are discussed. Interestingly, the optimal price for an on-line consultation may exceed that of a face-to-face consultation, but improvements in on-line consultation technology that reduce the optimal price can expand the size of the patient pool and thus increase optimal profits. Experts can charge a higher price on-line because as long as the on-line visit is more convenient than a face-to-face one, there is a segment of consumers who will value the on-line visit more. This implies that in the absence of a fixed cost for adopting the on-line channel, experts should always offer online consultations. As will also be shown, the quality of communication has more influence on the accuracy of an expert diagnosing a consumer whose state exhibits more uncertainty.

\section{Model Formulation}

The model is illustrated with the example of experts who sell professional opinions to a set of consumers. The consumers are uncertain about their individual states, which may be either good $(g)$ or bad $(b) .{ }^{1}$ Experts are differentiated with respect to the demand they face. An expert with index $i \in\{1, \ldots, N\}$ has a potential consumer base of size $D^{i}$. Consumers are differentiated with respect to the prior likelihood of being in a bad state. Within each potential consumer base, the prior probability of the bad state $(\alpha)$ is uniformly distributed among consumers between zero and one. Consumers incur a normalized unit loss if their state is bad, whereas no loss occurs if their state is good. In other words, if consumers knew with certainty that they were in the state, they would pay up to 1 in order to avoid the unit loss. Each consumer needs to decide whether to consult an expert for a possible treatment or do nothing. An expert suggests a treatment for the consumer upon diagnosing the bad state. If the diagnosis is correct, the consumer can avoid the associated unit loss by taking the suggested treatment. The cost of treatment to the consumer is less than the unit loss and is denoted by $c_{t}\left(0<c_{t}<1\right)$. Consumers may take the treatment only with the approval of an expert.

Experts can inspect consumers either face-to-face $(f)$ or on-line $(o)$. Consumers incur the transaction cost $t_{j}$ when they visit an expert via channel $j \in\{f, o\}$. The transaction cost on the face-to-face channel $\left(t_{f}\right)$ represents the cost of search and travel, the opportunity cost of time, and the implicit cost of inconvenience, whereas the transaction cost on the on-line channel $\left(t_{o}\right)$ represents the difficulty of gathering, storing, and sending personal information to the expert electronically. Since the latter is either negligible or has been decreasing steadily in recent years, $t_{o}$ is expected to be smaller than $t_{f}$.

A disadvantage of the on-line channel is that experts are likely to receive less information about consumers' problems when inspecting from a distance. 
For example, it is very hard, if not impossible, to diagnose a patient on-line if a physical examination is necessary. However, in some cases the expert may receive more information via the on-line channel than face to face, especially if the lack of direct physical contact with the expert makes the consumer feel more comfortable about sharing personal information (e.g., sexual problems). The quality of the information obtained via each channel is measured by the number of signals. The more signals the expert receives on a channel, the higher is the quality of communication on that channel. ${ }^{2}$ The number of signals transmitted via channel $j \in\{f, o\}$ is denoted by $n_{j}$. One objective of this research is to understand the role of the quality of communication (measured by $n_{j}$ ) on experts' optimal strategies.

Each signal an expert receives is drawn independently from the cumulative probability distribution $\Phi_{g}$ if the state is good and $\Phi_{b}$ if the state is bad. $\Phi_{g}$ and $\Phi_{b}$ are assumed to be normal distributions with variance $\sigma^{2}$ and means $\mu_{g}$ and $\mu_{b}$, respectively, where $\mu_{g}>\mu_{b}$. An expert aggregates all $n_{j}$ signals and chooses the cutoff aggregate signal $s_{j}$ such that, if the aggregate signal exceeds $s_{j}$, the expert concludes that the consumer is in the good state, and otherwise says that the consumer is in the bad state. If the null hypothesis is that the consumer is in the good state, the expert commits a Type I error by saying that a consumer is in the bad state when in fact the consumer is in the good state (rejecting the null hypothesis when it is true). The expert commits a Type II error by saying that the consumer is in the good state when the consumer is actually in the bad state (accepting the null hypothesis when it is false). The costs of Type I and Type II errors are denoted by $c_{1}$ and $c_{2}$, respectively. Note that the cost of a Type II error can be much higher than the cost of a Type I error, especially in health care. Experts aim to choose a cutoff aggregate signal $s_{j}$ that minimizes the expected cost of Type I and Type II errors.

Another technology parameter of interest is ease of use, which affects the time needed for each on-line consultation. An expert can work for at most $k$ hours. A normalized face-to-face consultation takes 1 hour, while an on-line consultation takes $\tau$ hours. One would expect $\tau$ to be less than 1 given the absence of physical inspection and the ability of the expert to schedule more consultations because of the asynchronous nature of on-line communication. Also, easy-to-use on-line consultation technologies would require less time for each expert-consumer interaction. Thus, an expert can handle at most $k$ consumers face-to-face and $k / \tau$ consumers on-line. The focus here is on experts who are in high demand (e.g., acclaimed doctors) such that the capacity constraint always binds. ${ }^{3}$

Taking into account the capacity constraint and the distribution of consumer states, experts select their optimal diagnosis policy and price for each channel they choose to serve. After observing the experts' channel strategies, consumers decide whether to consult an expert in maximizing their expected utility. If the selected expert utilizes both channels, consumers also choose whether to visit the expert face-to-face or on-line. A complete list of notations is provided in the appendix. 


\section{Analysis}

\section{Cost and Value of Face-to-Face and On-line Consultations}

$\Phi_{g j}$ and $\Phi_{b j}$ are denoted as the joint cumulative probability distributions of a mean of $n_{j}$ signals when a consumer is in the good and bad states, respectively. Given the underlying normal distribution of each signal, $\Phi_{g j}$ and $\Phi_{b j}$ are distributed normally with variance $\sigma^{2} / n_{j}$ and means $\mu_{g}$ and $\mu_{b}$, respectively. If the prior likelihood of the bad state is $\alpha$, then the likelihood that an expert will say a consumer is in the bad state when in fact the consumer is in the good state is $(1-\alpha) \Phi_{g j}\left(s_{j}\right)$. Similarly, the likelihood that the expert will say the consumer is in the good state when the consumer is actually in the bad state is $\alpha\left(1-\Phi_{b j}\left(s_{j}\right)\right)$. Thus, given the prior likelihood of the bad state $\alpha$, the expected cost of Type I and Type II errors for a diagnosis provided via channel $j$ equals

$$
C\left(s_{j}, \alpha\right)=(1-\alpha) \Phi_{g j}\left(s_{j}\right) c_{1}+\alpha\left(1-\Phi_{b j}\left(s_{j}\right)\right) c_{2} \text {. }
$$

The value of a consultation depends on how much it improves a consumer's expected utility. A customer who does not consult an expert can maintain the unit utility only if the state is good, and therefore can expect a utility of $\operatorname{Pr}($ state $=g)=1-\alpha$. The diagnosis of the expert delivered via channel $j$ is denoted by $d_{j}$. If the expert diagnoses the bad state, the consumer takes the treatment, incurs $c_{t}$, and maintains the unit utility. If the expert diagnoses the good state, the consumer cannot take the treatment regardless of the prior likelihood of the bad state, and maintains the unit utility with probability $\operatorname{Pr}\left(\right.$ state $\left.=g \mid d_{j}=g\right)$. In other words, if the expert diagnoses the good state, the consumer loses the unit utility with the probability of the state actually being bad given the diagnosis $\left(1-\operatorname{Pr}\left(\right.\right.$ state $\left.\left.=g \mid d_{j}=g\right)\right)$. Upon obtaining the consultation via channel $j$ (and incurring the transaction cost $t_{j}$ ), the consumer's expected utility $E\left(U \mid d_{j}\right)$ equals

$$
\begin{aligned}
E\left(U \mid d_{j}\right) & =\operatorname{Pr}\left(d_{j}=b\right)\left(1-c_{t}\right)+\operatorname{Pr}\left(d_{j}=g\right) \operatorname{Pr}\left(\text { state }=g \mid d_{j}=g\right)-t_{j} \\
& =\operatorname{Pr}\left(d_{j}=b\right)\left(1-c_{t}\right)+\operatorname{Pr}\left(\text { state }=g, d_{j}=g\right)-t_{j} .
\end{aligned}
$$

Rearranging the terms, we obtain

$$
\begin{aligned}
E\left(U \mid d_{j}\right)=\operatorname{Pr}(\text { state } & =g)+\operatorname{Pr}(\text { state }=b) \operatorname{Pr}\left(d_{j}=b \mid \text { state }=b\right) \\
& -\operatorname{Pr}\left(d_{j}=b\right) c_{t}-t_{j} .
\end{aligned}
$$

The value of a consultation equals the expected utility post consultation $\left(E\left(U \mid d_{j}\right)\right)$ less the expected utility with no consultation with the expert $(1-\alpha)$ : 


$$
\begin{aligned}
V_{j}\left(s_{j}, \alpha\right)= & \operatorname{Pr}(\text { state }=b) \operatorname{Pr}\left(d_{j}=b \mid \text { state }=b\right)-\operatorname{Pr}\left(d_{j}=b\right) c_{t}-t_{j} \\
& =\alpha \Phi_{b j}\left(s_{j}\right)\left(1-c_{t}\right)-(1-\alpha) \Phi_{g j}\left(s_{j}\right) c_{t}-t_{j} .
\end{aligned}
$$

Equation (2) indicates that the value of a consultation increases with both the prior likelihood of the bad state and the accuracy of the expert in correctly predicting the bad state. On the other hand, the value decreases with the cost of the treatment itself and with the likelihood that the expert mistakenly suggests unnecessary treatment. That is, the importance of a misdiagnosis compounds when the cost of treatment is high. Because the expert is more likely to misdiagnose a consumer's state when $n_{j}$ is low, and since one would expect $n_{0}$ to be less than $n_{f}$ in most cases because of the absence of physical inspection, the expert is less likely to utilize on-line consultations when the stakes are high for the consumer.

Equation (2) also implies that a consumer's valuation for a consultation increases with the likelihood of the bad state ( $\alpha$ ), but more so for the channel from which the expert receives fewer signals. This is because the quality of the communication medium does not have much effect on the value of the service when there is little or no uncertainty about the consumer's state. Lemma 1 states this finding for $n_{f}>n_{0}$.

Lemma 1. The values of both face-to-face and on-line consultations increase with the likelihood of the bad state. However, when $n_{f}>n_{0}$, the value of an on-line consultation increases at a higher rate than the value of a face-to-face consultation. Conversely, as the good state becomes more likely, the value of an on-line consultation decreases at a slower rate than the value of a face-toface consultation.

\section{Optimal Diagnosis and Channel Strategies}

In order to get the highest return for labor, each expert selects the group of customers who place the highest value on the service provided and sets a diagnosis policy. For an expert to utilize the Internet, some of these customers should prefer to be served on-line. Intuitively, on-line consultations should be more appealing when the face-to-face transaction cost $\left(t_{f}\right)$ is greater than its on-line counterpart $\left(t_{o}\right)$. However, as shown in Proposition 1, there is a segment of consumers that prefer an on-line consultation to a face-to-face one as long as $t_{f}>t_{0}$. This leads experts to always offer the on-line service. The proof is in the appendix.

Proposition 1. Given $t_{f}>t_{0}$, there is a segment of consumers with high $\alpha$ who value an on-line consultation more than a face-to-face one.

The optimal channel and diagnosis policies are determined simultaneously. Corollary 1 establishes that whenever an expert offers both services, consumers with a high $\alpha$ prefer the on-line service, while those with a low $\alpha$ 
prefer the face-to-face one. For example, consider the special case $\alpha=1$ where both the consumer and the expert know with certainty that the state is bad. Since there is no room for diagnostic error in this special case, and given that the consumer can obtain the same treatment on both channels, the channel preference will be based solely on convenience, and the consumer will prefer on-line over face-to-face consultation. However, as the prior likelihood of the bad state decreases, the probability of a misdiagnosis increases at a faster rate on-line than face-to-face if the quality of communication is lower on this channel $\left(n_{f}>n_{0}\right)$, and so the consumer may prefer a face-to-face consultation below a threshold $\alpha$ value. The proof of Corollary 1 is also in the appendix.

Corollary 1. Given $t_{f}>t_{o}$, whenever an expert offers both services (which happens only when $n_{f}>n_{o}$ ), consumers with high (low) $\alpha$ values prefer the on-line (face-to-face) service.

Patients with chronic health problems illustrate this point. On-line consultations are reported to be especially useful for patients who have a chronic condition such as diabetes, asthma, hypertension, or heart problems [18]. Therefore, these services can be successfully offered to chronic disease populations for whom prescription refills, appointments, and laboratory tests are most frequent, rather than to healthy populations with intermittent illnesses requiring diagnostic evaluation. According to Harris Interactive, 71 percent of American adults who have Internet access prefer to get new prescriptions on-line for medications they already take [34]. Clearly, patients do not prefer to physically visit the doctor's office when their condition and the necessary treatment are known a priori.

According to Corollary 1, each expert serves consumers with high $\alpha$ values as long as treatment can be provided both face-to-face and on-line. The lowest $\alpha$ value among the consumers served by expert $i$ is denoted by $\underline{\alpha}^{i}$. In addition, assuming that expert $i$ offers consultations on both channels at optimality, the cutoff $\alpha$ value above which the expert serves on-line is denoted by $\bar{\alpha}^{i}$. Since the expert works at capacity:

$$
D^{i}\left(1-\bar{\alpha}^{i}\right) \tau+D^{i}\left(\bar{\alpha}^{i}-\underline{\alpha}^{i}\right)=k .
$$

According to Corollary 2, the consumer with $\alpha=\underline{\alpha}^{i}$ prefers a face-to-face consultation when both channels are used. Therefore, the most the expert can charge this consumer for a face-to-face consultation while working at capacity is $p_{f}^{i}=V_{f}\left(\underline{\alpha}^{i}\right)$.

Taking as given the costs of Type I and Type II errors and the distribution of prior likelihoods of the bad state, expert $i$ decides which consumers to serve on-line/face-to-face and which diagnosis policy to employ on each channel. This decision, in turn, determines the price that can be charged for the two types of services. By definition, the on-line and face-to-face prices of the expert make the consumer with $\alpha=\bar{\alpha}^{i}$ indifferent between obtaining a face-to-face and an on-line consultation: 


$$
V_{f}\left(s_{f}, \bar{\alpha}^{i}\right)-p_{f}^{i}=V_{o}\left(s_{o}, \bar{\alpha}^{i}\right)-p_{o}^{i} .
$$

Plugging in the value of $p_{f}^{i}$, we have

$$
p_{o}^{i}=V_{o}\left(s_{o}, \bar{\alpha}^{i}\right)-V_{f}\left(s_{f}, \bar{\alpha}^{i}\right)+V_{f}\left(s_{f}, \underline{\alpha}^{i}\right) .
$$

Given the expected per diagnosis cost of Type I and Type II errors in Equation (1) and the linear relationship between $\underline{\alpha}^{i}$ and $\bar{\alpha}^{i}$ in Equation (3), the expert's problem can be stated as that of choosing the customer's $\bar{\alpha}^{i}$ and $\underline{\alpha}^{i}$ and diagnosis policies $s_{f}$ and $s_{o}$ that maximize total net profit:

$$
\begin{gathered}
\operatorname{Max}_{\bar{\alpha}^{i}, \underline{\alpha}^{i}, s_{f}, s_{o}} \Pi^{i}=D^{i}\left[\left(1-\bar{\alpha}^{i}\right) p_{o}^{i}+\left(\bar{\alpha}^{i}-\underline{\alpha}^{i}\right) p_{f}^{i}-\int_{\underline{\alpha}^{i}}^{\bar{\alpha}^{i}} C\left(s_{f}, \alpha\right) d \alpha-\int_{\bar{\alpha}^{i}}^{1} C\left(s_{o}, \alpha\right) d \alpha\right] \\
\text { s.t. } D^{i}\left(1-\bar{\alpha}^{i}\right) \tau+D^{i}\left(\bar{\alpha}^{i}-\underline{\alpha}^{i}\right)=k .
\end{gathered}
$$

Since the optimal $\bar{\alpha}^{i}$ determines the optimal $\underline{\alpha}^{i}$, it is necessary to maximize with respect to $\bar{\alpha}^{i}$. The following equality is obtained from the first-order condition for $\bar{\alpha}^{i *}$ :

$$
\begin{gathered}
\left(1-\bar{\alpha}^{i^{*}}\right)\left(\frac{\partial V_{o}}{\partial \bar{\alpha}^{i}}-\frac{\partial V_{f}}{\partial \bar{\alpha}^{i}}\right)+\left(1-\underline{\alpha}^{i^{*}}\right) \frac{\partial V_{f}}{\partial \bar{\alpha}^{i}}(1-\tau)=V_{o}\left(s_{o}^{*}, \bar{\alpha}^{i^{*}}\right)-V_{f}\left(s_{f}^{*}, \bar{\alpha}^{i^{*}}\right) \\
+(1-\tau) V_{f}\left(s_{f}^{*}, \underline{\alpha}^{i^{*}}\right)-C\left(s_{o}^{*}, \bar{\alpha}^{i^{*}}\right)+C\left(s_{f}^{*}, \bar{\alpha}^{i^{*}}\right)-(1-\tau) C\left(s_{f}^{*}, \underline{\alpha}^{i^{*}}\right) .
\end{gathered}
$$

Similarly, the expert sets the optimal cutoff policies $s_{f}^{*}$ and $s_{o}^{*}$ according to the following equalities obtained from the first-order conditions for $s_{f}^{*}$ and $s_{0}^{*}$, respectively:

$$
\begin{gathered}
D^{i}\left(1-\bar{\alpha}^{i^{*}}\right)\left[-\frac{\partial V_{f}\left(s_{f}^{*}, \bar{\alpha}^{i^{*}}\right)}{\partial s_{f}}+\frac{\partial V_{f}\left(s_{f}^{*}, \underline{\alpha}^{i^{*}}\right)}{\partial s_{f}}\right]+D^{i}\left(\bar{\alpha}^{i^{*}}-\underline{\alpha}^{i^{*}}\right) \frac{\partial V_{f}\left(s_{f}^{*}, \underline{\alpha}^{i^{*}}\right)}{\partial s_{f}} \\
=\frac{\partial C\left(s_{f}^{*}, \underline{\alpha}^{i^{*}}\right)}{\partial s_{f}} \\
D^{i}\left(1-\bar{\alpha}^{i^{*}}\right) \frac{\partial V_{o}\left(s_{o}^{*}, \bar{\alpha}^{i^{*}}\right)}{\partial s_{o}}=\frac{\partial C\left(s_{o}^{*}, \bar{\alpha}^{i^{*}}\right)}{\partial s_{o}} .
\end{gathered}
$$

Equation (4) states that, at the interior optimum, the marginal benefit and the marginal cost of raising $\bar{\alpha}^{i}$ are equal. A unit increase in $\bar{\alpha}^{i}$ leads to a unit decrease in the number of on-line consultations and a $\tau$ increase in the number of face-to-face consultations. Thus, the total number of consultations served 

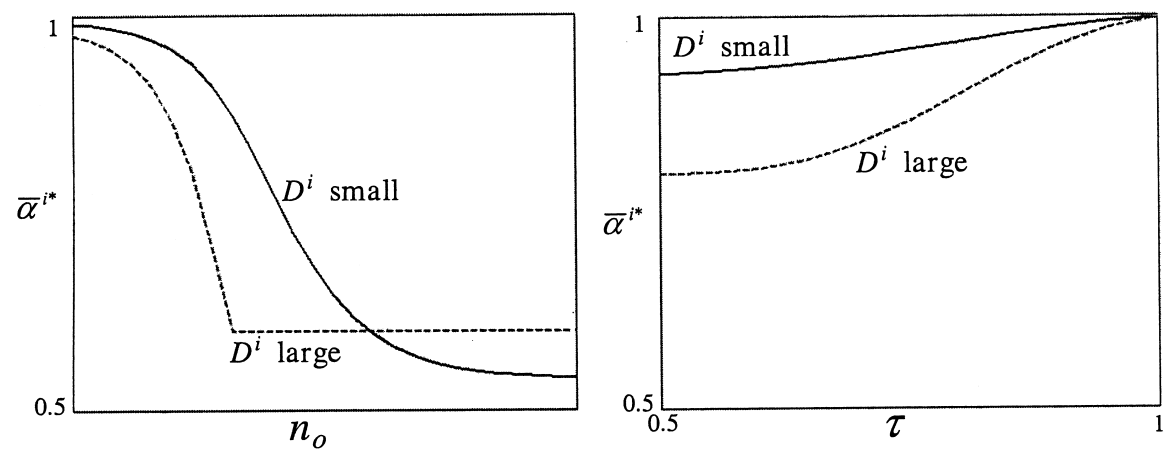

Figure 1. Optimal Channel Mix as a Function of Market Demand and the Online Channel's Quality of Communication $\left(n_{0}\right)$ and ease of use $(\tau)$

by expert $i$ decreases by $1-\tau$. Catering to a smaller consumer segment allows experts to serve those who have a higher value for their services. Consequently, the expert can charge $\left(\left(\partial V_{f}\left(\underline{\alpha}^{i *}\right)\right) / \partial \bar{\alpha}^{i *}\right)(1-\tau)$ more to all $D^{i}\left(1-\underline{\alpha}^{i *}\right)$ customers. Further, the expert can charge $\left(\partial V_{o}\left(\bar{\alpha}^{i *}\right) / \partial \bar{\alpha}^{i}\right)-\left(\partial V_{f}\left(\bar{\alpha}^{i *}\right) / \partial \bar{\alpha}^{i}\right)$ more to $D^{i}\left(1-\bar{\alpha}^{i *}\right)$ on-line customers. On the other hand, the expert loses $D^{i}\left[V_{o}\left(\bar{\alpha}^{i *}\right)-V_{f}\left(\bar{\alpha}^{i *}\right)+\right.$ $\left.V_{f}\left(\underline{\alpha}^{i *}\right)\right]$ at the margin due to raising $\bar{\alpha}^{i *}$ and selling fewer on-line consultations, and can only make up $D^{i} \tau V_{f}\left(\underline{\alpha}^{i *}\right)$ by selling additional face-to-face consultations. Because fewer consultations are served, the total cost of diagnosis errors committed reduces by $D^{i}\left[C\left(s_{0}^{*}, \bar{\alpha}^{i *}\right)-C\left(s_{f}^{*}, \bar{\alpha}^{i *}\right)+(1-\tau) C\left(s_{f}^{*}, \underline{\alpha}^{i *}\right)\right]$.

Figures 1 and 2 illustrate the intuitions behind Equation (4). Figure 1(a) shows that the utilization of the on-line channel increases with $n_{0}$. However, a relatively high demand increases the time benefit of the on-line consultation and thereby the utilization of the Internet. Figure 1(b) shows that an expert should handle more consumers on-line as $\tau$ decreases. Here again the time benefit of on-line consultations becomes an important factor when the expert has a relatively high demand. The expert may start serving less on-line below a certain $\tau$ value because a low $\tau$ substantially increases the expert's capacity and limits his ability to charge a high price in the absence of price discrimination.

When $\tau<1$, the increased utilization of the on-line channel due to a high $n_{o}$ also increases the number of consumers served and thereby puts a downward pressure on prices (see Figure 2(a)). If the demand and $n_{o}$ are sufficiently large, the expert can operate entirely via the on-line channel. In this case a further increase in $n_{0}$ does not increase the number of consumers served, and the expert raises the on-line price to take advantage of further improvements in technology. Figure 2(b) shows that the optimal on-line price is higher than the face-to-face price when the two types of consultations take at about the same time. As $\tau$ decreases, experts find the on-line channel more attractive and lower their on-line prices to serve more consumers via the Internet. Figures 2(c) and 2(d) indicate that profits increase with improvements in on-line consultation technology in terms of both quality of communication $\left(n_{0}\right)<<0$ rather than 0 ? $>>$ and ease of use $(\tau)$. However, since profits increase at a 

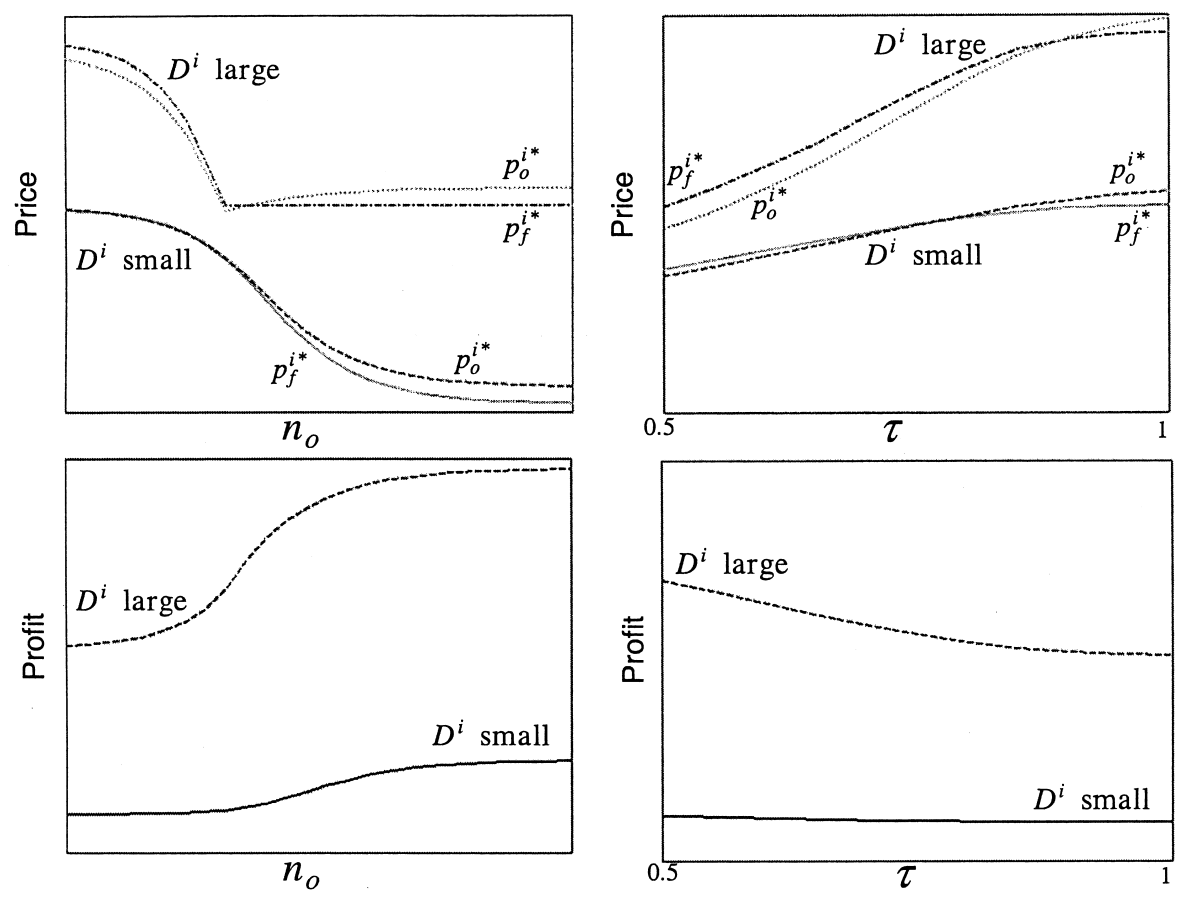

Figure 2. Optimal Prices and Profits as a Function of Market Demand, the Online Channel's Quality of Communication $\left(n_{0}\right)$ and ease of use $(\tau)$

higher rate when capacity is more limited relative to the demand, one may conjecture that the medical experts who are most pressed for time will benefit more from on-line consultations and should seriously consider adoption as technology improves.

Proposition 2. While an expert may expect a higher return from on-line service, the optimal on-line price decreases as on-line consultation technology becomes easier to use. The higher the demand relative to capacity, the more an expert benefits from improvements in quality of communication and ease of use.

Equations (5) and (6) state that the marginal benefit and the marginal cost of raising $s_{f}$ and $s_{o}$ are equal, respectively. By incrementally increasing $s_{f}$ an expert can charge $-\left(\partial V_{f}\left(\bar{\alpha}^{i}\right) / \partial s_{f}\right)+\left(\partial V_{f}\left(\underline{\alpha}^{i}\right) / \partial s_{f}\right)$ more for on-line service to $D^{i}\left(1-\bar{\alpha}^{i *}\right)$ customers. Similarly, the expert can charge $\partial V_{f}\left(\underline{\alpha}^{i}\right) / \partial s_{f}$ more for face-to-face service to $D^{i}\left(\bar{\alpha}^{i *}-\underline{\alpha}^{i *}\right)$ customers. The change in the expected cost of Type I and Type II errors in this case is $\partial C\left(s_{f}^{*}, \underline{\alpha}^{i *}\right) / \partial s_{f}$, which should be equal to the expected gain of incrementally increasing $s_{f}$. A change in $s_{o}$ does not affect the expert's face-to-face price, which is only a function of $s_{f}$. By incrementally increasing $s_{0}$, the expert can charge $\partial V_{o}\left(\bar{\alpha}^{i}\right) / \partial s_{0}$ more for on-line service to $D^{i}\left(1-\bar{\alpha}^{i *}\right)$ customers. Such a change affects the expected cost of Type I and Type II errors by $\partial C\left(s_{0}{ }^{*}, \underline{\alpha}^{i *}\right) / \partial s_{0},<<$ LHS should be $s_{o}$ rather than $s_{0}$ ? >> 


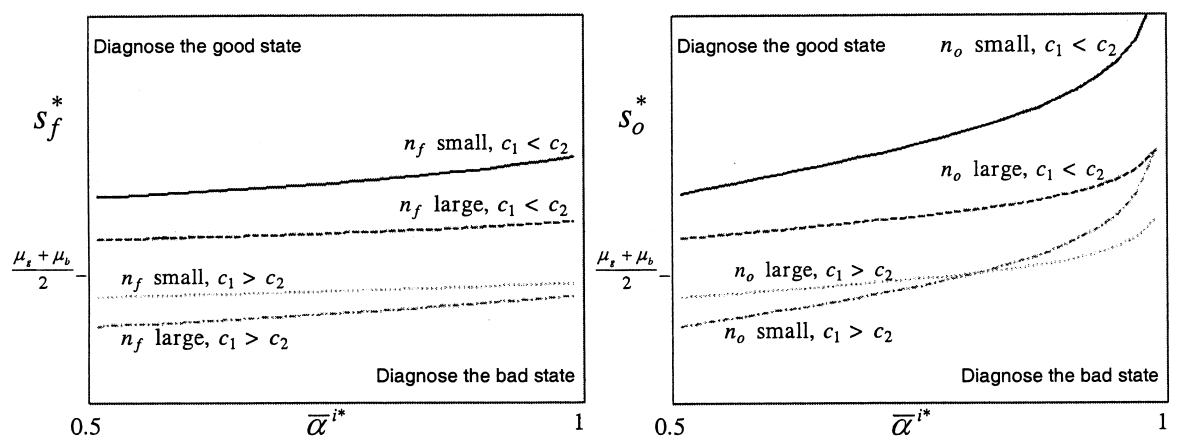

Figure 3. Diagnosis Policies as a Function of $\bar{\alpha}^{i *}, n_{i}$ and $c_{1} / c_{2}:$ (a) faceto-face and (b) online

Figure 3 illustrates the intuitions behind Equations (5) and (6). ${ }^{5}$ First, the optimal cutoff aggregate signal increases with the relative costs of Type II versus Type I errors. Since misdiagnosing a consumer with the good state is costlier than misdiagnosing a consumer with the bad state when $c_{1}<c_{2}$, and vice versa, consumers are more likely to be diagnosed with the bad state when $c_{1}<c_{2}$ than otherwise. In part because of malpractice suits against doctors, this behavioral pattern is prevalent in the U.S. health care sector and is known as physician-induced demand in health-care economics. Second, as the prior likelihood of the bad state increases, experts rely more on this likelihood than the observed signals about the state, and thus they increase their cutoff aggregate signals and diagnose the bad state more frequently. In other words, the same aggregate signal may lead an expert to diagnose different states depending on the prior probability of the bad state. And third, when $c_{1}<c_{2}$, less information (smaller $n_{j}$ ) leads experts to diagnose the bad state more often as they try to be on the safe side, but at the expense of diagnostic accuracy. This observation implies that by restricting the flow of information between experts and consumers, the on-line environment can exacerbate the problem of unnecessary prescription of treatments.

\section{Discussion and Conclusions}

Past research on telemedicine mainly addresses the question "Can we do this?" and leaves "Should we do this?" unanswered [26]. The latter question should be tackled from the perspective of the providers and take account of their physical operations. The study of optimal channel-management strategies for experts provided here, while significant in its own right, is uniquely positioned at the interface of the literatures on telemedicine, multi-channel management, and expert services.

The diagnosis problem confronting experts has been modeled as that of selecting the state of a consumer under uncertainty, where diagnostic accuracy depends critically on the quality of communication in a consultation. Experts face different levels of demand, are capacity-constrained, and can consult both 
face-to-face and on-line. Consumers maximize expected surplus, while experts maximize profit. The insights obtained from the model offer several practical implications that can help experts to set the extent of their on-line offerings and decide whom to serve on-line within their specialty given their patient portfolio. For example, according to a recent Harris Interactive survey, most physicians have serious reservations about consulting on-line because, among other things, of concerns about reimbursement [34]. The present research shows that experts will always be able to find consumers who are willing to pay more for an on-line service than a face-to-face one. Interestingly, physicians may still have to charge a lower price on-line at optimality in order to serve a larger patient pool. As on-line consultation technology improves, physicians may have to charge even less on-line but would earn more, especially if they are facing a high demand and therefore are pressed for time. Also, medical experts should target their on-line services to consumers with more certain health conditions, because the potentially negative effect of communication quality would be more pronounced when inspecting patients with more uncertain state expectations.

Of course, the stylized model considered here applies only to certain situations. For example, the preceding discussion only considers the case where getting an on-line consultation is more convenient than getting a face-to-face one $\left(t_{f}>t_{0}\right)$, disregarding the medical conditions that render obtaining and sending personal information prohibitively costly for patients. Still, the analysis covers many situations because of the recent advances in telemedicine technologies that ease the collection and transfer of patient information. Today's technologies enable doctors to monitor their patients' every heartbeat as they go about their daily activities. Even in the most remote locations, sensors connected to patients can beam images and signals to medical experts at distant centers for real-time consultations, diagnoses, and monitoring. Second, it was assumed that the fixed cost of adopting on-line consultations is zero because physicians only need a basic Internet connection to offer these services (e.g., using Medem's secure messaging suite of tools), and that the cost of an Internet connection is negligible compared to many other cost items in health care. Certain types of consultations, however, may require special technical equipment. In psychiatry, for example, videoconferencing equipment may be necessary to get a good sense of patients' problems. In such circumstances, the optimal investment in on-line consultation technology would be another important decision. It could be investigated in future research by extending the current model in a way that would associate the amount of investment in the technology to the quality of communication.

\section{NOTES}

1. The bad state can be thought of as having a specific disease or sickness. Thus, the expert's job is to diagnose whether the consumer does indeed have this adverse condition.

2. In real life, some observations about the state can be more informative than others. The notion of equally informative signals used here is not restrictive, because more informative observations may be considered as equivalent to multiple equally 
(less) informative signals in terms of their possible effect on the variance of the posterior probability of the bad state.

3. These results may apply to physicians in general, given the net shortage of doctors in the United States. See, for example, www.ama-assn.org/ama/pub/category/15241.html. The qualitative results do not change when the capacity constraint does not bind.

4. Figures 1 and 2 were drawn using the following parameter values: $\mu_{b}=0$, $\mu_{g}=10, \sigma=10, c_{1}=1, c_{2}=5, c_{t}=0.05, t_{f}=0.005, t_{o}=0, n_{f}=100, k=12$ (hours), $D_{\text {small }}^{i}=$ 30 , and $D_{\text {large }}^{i}=40$. Figures 1(a), 2(a), and 2(c) were drawn assuming that an on-line consultation takes less time than a face-to-face one $(\tau=0.75<1)$, whereas Figures 1(b), 2(b), and 2(d) were drawn assuming that an on-line consultation provides less information than a face-to-face one $\left(n_{o}=40<n_{f}=100\right)$.

5. Figures 3(a) and 3(b) were drawn using the following parameter values: $\mu_{b}=0, \mu_{g}=10, \sigma=10, c_{t}=0.05, t_{f}=0.005, t_{o}=0, \tau=0.75, k=12$ (hours), and $D^{i}=40$. $c_{1}<c_{2}$ implies $c_{1}=1$ and $c_{2}=5$, and conversely. Small (large) $n_{j}$ implies $n_{j}=40\left(n_{j}=\right.$ 40) for $j \in\{f, o\}$.

\section{REFERENCES}

1. Alger, I., and Salanié, F. A theory of fraud and overtreatment in expert markets. Journal of Economics \& Management Strategy, 15, 4 (December 2006), 853-881.

2. Bakos, J.Y. Reducing buyer breach costs: Implications for electronic marketplaces. Management Science, 43, 12 (December 1997), 1676-1692.

3. Bakos, J.Y., and Brynjolfsson, E. Bundling information goods: Pricing, profits, and efficiency. Management Science, 45, 12 (December 1999), 1613-1630.

4. Balasubramanian, S. Mail versus mall: A strategic analysis of competition between direct marketers and conventional retailers. Marketing Science, 17, 3 (1998) 181-195.

5. Bartels, R.; Fiebig, D.; and Soest, A. Consumers and experts: An econometric analysis of the demand for water heaters. Empirical Economics, 31, 2 (May 2006), 369-391.

6. Bouckaert, J., and Degryse, H. Price competition between an expert and a non-expert. International Journal of Industrial Organization, $18<<$ issue and/ or season $>>$ (2000), 901-923.

7. Byers, R.E., and Lederer, P.J. Retail bank services strategy: A model of traditional, electronic, and mixed distribution choices. Journal of Management Information Systems, 18, 2 (fall 2001), 133-156.

8. Carrns, A. Medem to enable physicians to charge for online visits. Wall Street Journal, February 25, 2003, <<p.?>>.

9. Chen Y., and Png, I. Information goods pricing and copyright enforcement: Welfare analysis. Information Systems Research, 14, 1 (March 2003), 107-123.

10. Chiang, W.K.; Chhajed, D.; and Hess, J.D. Direct marketing, indirect profits: A strategic analysis of dual-channel supply-chain design. Management Science, 49, 1 (January 2003), 1-20.

11. Committee on the Quality of Health Care in America, Institute of Medicine. Crossing the Quality Chasm: A New Health System for the 21st Century. Washington, DC: National Academy Press, 2001. 
12. Darby, M., and Karni, E. Free competition and the optimal amount of fraud. Journal of Law and Economics, 16, 1 (April 1973), 67-88.

13. Dewan, R.; Freimer, M.; and Seidmann, A. Organizing distribution channels for information goods on the Internet. Management Science, 46, 4 (April 2000), 483-495.

14. Edlin, M. Joint ventures and coalitions drive the e-prescribing bandwagon. Managed Healthcare Executive, 12, 12 (December 2002), 30-31.

15. Emons, W. Credence goods and fraudulent experts. RAND Journal of Economics, 28 <<issue>> (spring 1997), 107-119.

16. Emons, W. Credence goods monopolists. International Journal of Industrial Organization, 19 <<issue>> (March 2001), 375-389.

17. Fox, S., and Fallows, D. Internet Health Resources (www.pewinternet. org/pdfs/PIP_Health_Report_July_2003.pdf).

18. Freudenheim, M. Digital Rx: Take two aspirins and e-mail me in the morning. New York Times, March 2, 2005, <<p.?>>.

19. Hailey, D.; Roine, R.; and Ohinmaa, A. Systematic review of evidence for the benefits of telemedicine. Journal of Telemedicine and Telecare, 8 (2002), Supplement 1,1-7.

20. Hailey, D.; Roine, R.; and Ohinmaa, A. Study quality and evidence of benefit in recent assessments of telemedicine. Journal of Telemedicine and Telecare, 10, 6 (December 2004) 318-324.

21. Hann, I., and Terwiesch, C. Measuring the frictional costs of online transactions: The case of a name-your-own-price channel. Management Science, 49, 11 (November 2003) 1563-1579.

22. Hitt, L.M., and Frei, F.X. Do better customers utilize electronic distribution channels? The case of PC banking. Management Science, $48<<$ issue >> (June 2002), 732-748.

23. King, R.C.; Sen, R.; and Xia, M. Impact of Web-based e-commerce on channel strategy in retailing. International Journal of Electronic Commerce, 8, 3 (spring 2004), 103-130.

24. Lal, R., and Sarvary, M. When and how is the Internet likely to decrease price competition? Marketing Science, 18, 4 (1999), 485-503.

25. Lynch, J., and Ariely, D. Wine online: Search costs affect competition on price, quality, and distribution. Marketing Science, 19, 1 (winter 2000), 83-103.

26. Mair, F.S.; Haycox, A.; May, C.; and Williams, T. A review of telemedicine cost-effectiveness studies. Journal of Telemedicine and Telecare, 6 (2000), Supplement 1, 38-40.

27. Ozdemir, Z.D.; Akcura, M.T.; and Altinkemer, K. Second opinions and online consultations. Decision Support Systems, 42, 3 (December 2006), 1747-1758.

28. Pesendorfer, W., and Wolinsky, A. Second opinions and price competition: Inefficiency in the market for expert advice. Review of Economic Studies, $70<<$ issue $>>$ (April 2003), 417-437.

29. Pitchik, C., and Schotter, A. Honesty in a model of strategic information transmission. American Economic Review, 77 <<issue>> (December 1987), 1032-1036. 
30. Riggins, F.J. A multichannel model of separating equilibrium in the face of the digital divide. Journal of Management Information Systems, 21, 2 (fall 2004), 161-179.

31. Sarvary, M. Temporal differentiation and the market for second opinions. Journal of Marketing Research, 39 <<issue>> (February 2002), 129-136.

32. Steinfield, C.; Bouwman, H.; and Adelaar, T. The dynamics of click-andmortar electronic commerce: Opportunities and management strategies. International Journal of Electronic Commerce, 7, 1 (fall 2002), 93-119.

33. Taylor, C.R. The economics of breakdowns, checkups and cures. Journal of Political Economy, 103 <<issue >> (February 1995), 53-74.

34. Taylor, H., and Leitman, R. (eds.). Health Care News (www.harrisinteractive.com/news/newsletters/healthnews/HI_HealthCareNews2002V012 Iss08.pdf).

35. Terry, K. Online "visits" insurers pay. Medical Economics, 83, 17 (September 1, 2006), 30-34.

36. Viswanathan, S. Competing across technology-differentiated channels: The impact of network externalities and switching costs. Management Science, 51, 3 (March 2005), 483-496.

37. Wolinsky, A. Competition in a market for informed experts' services. RAND Journal of Economics, 24 <<issue >> (fall 1993), 380-398.

38. Zettelmeyer, F. Expanding to the Internet: Pricing and communications strategies when firms compete on multiple channels. Journal of Marketing Research, 37 <<issue >> (August 2000), 292-308.

\section{Appendix}

\section{Proof of Proposition 1}

It is only necessary to show $V_{o}(\alpha)>V_{f}(\alpha)$ for some $\alpha \in[0,1]$, given $t_{f}>t_{o}$. Consider the consumer who is certain of being in the bad state $(\alpha=1)$. For this consumer $V_{o}(1)>V_{f}(1)$ when $t_{f}-t_{o}>\left(1-c_{t}\right)\left[\Phi_{b f}\left(s_{f}\right)-\Phi_{b o}\left(s_{o}\right)\right]$. But since the state is certain when $\alpha=1$, the expert always correctly diagnoses on both channels (i.e., $\left.\Phi_{b f}\left(s_{f}\right)=\Phi_{b o}\left(s_{o}\right)=1\right)$. Thus, as long as $t_{f}>t_{o}$, consumers who are very likely to be in the bad state derive more value from on-line consultations.

\section{Proof of Corollary 1}

The proof follows from Lemma 1 and Proposition 1. For $n_{o}>n_{f}, V_{o}(\alpha)>V_{f}(\alpha)$ for all $\alpha \in[0,1]$. In this case the expert offers only the on-line service. By Proposition 1, consumers with a very high $\alpha$ prefer the on-line service. By Lemma 1, when $n_{f}>n_{o}$, the on-line consultation loses its value faster than the face-to-face consultation as $\alpha$ decreases from 1. Consequently, consumers with lower $\alpha$ may prefer the face-to-face consultation if $f_{t}-t_{o}$ is sufficiently small, which should be the case for the expert to offer the face-to-face service. 


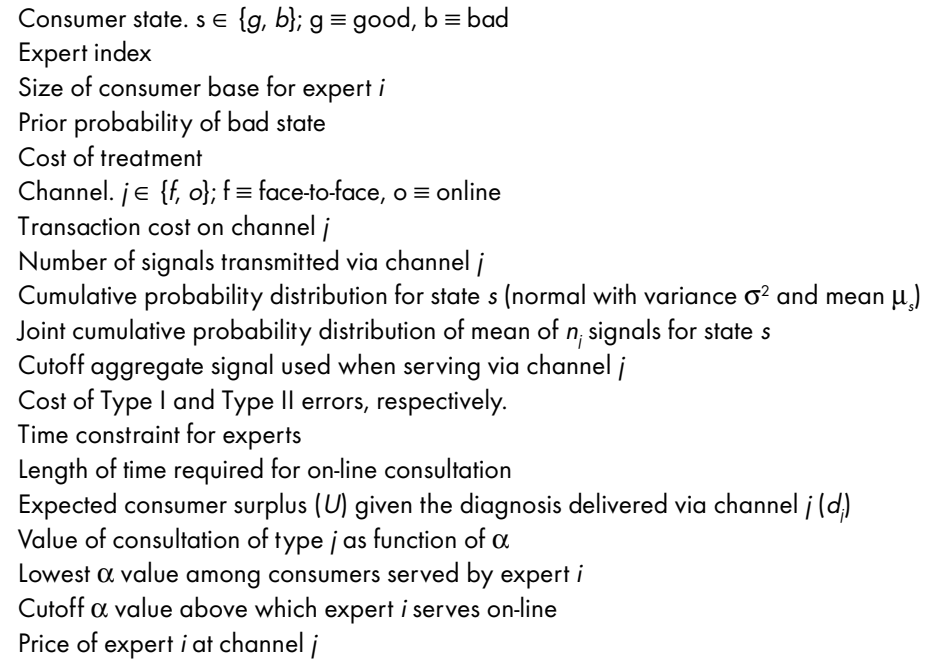

Table 1. Notations.<<need in-text citation / appendix?〉>

ZAFER D. OZDEMIR (ozdemir@muohio.edu) is an assistant professor at the Richard T. Farmer School of Business Administration, Miami University. He has a B.Sc. and an M.A. from Bogazici University and a Ph.D. from Purdue University. His research focuses on the economics of e-health and technology-mediated learning. 